\title{
Rays to renormalizations
}

by

\author{
Genadi LEVIN
}

Presented by Feliks PRZYTYCKI

Summary. Let $K_{P}$ be the filled Julia set of a polynomial $P$, and $K_{f}$ the filled Julia set of a renormalization $f$ of $P$. We show, loosely speaking, that there is a finite-to-one function $\lambda$ from the set of $P$-external rays having limit points in $K_{f}$ onto the set of $f$-external rays to $K_{f}$ such that $R$ and $\lambda(R)$ share the same limit set. In particular, if a point of the Julia set $J_{f}=\partial K_{f}$ of a renormalization is accessible from $\mathbb{C} \backslash K_{f}$ then it is accessible through an external ray of $P$ (the converse is obvious). Another interesting corollary is that a component of $K_{P} \backslash K_{f}$ can meet $K_{f}$ only in a single (pre-)periodic point. We also study a correspondence induced by $\lambda$ on arguments of rays. These results are generalizations to all polynomials (covering notably the case of connected Julia set $K_{P}$ ) of some results of Levin and Przytycki (1996), Blokh et al. (2016) and Petersen and Zakeri (2019) where it is assumed that $K_{P}$ is disconnected and $K_{f}$ is a periodic component of $K_{P}$.

\section{Introduction}

1.1. Polynomial external rays. Let $Q: \mathbb{C} \rightarrow \mathbb{C}$ be a non-linear polynomial considered as a dynamical system. Conjugating $Q$ if necessary by a linear transformation, one can assume without loss of generality that $Q$ is monic centered, i.e., $Q(z)=z^{\operatorname{deg}(Q)}+a z^{\operatorname{deg}(Q)-2}+\cdots$.

We briefly recall the necessary definitions (see e.g. [DH1, [CG], Mil0], [LS91] for details). The filled Julia set $K_{Q}$ of $Q$ is the complement $\mathbb{C} \backslash A_{Q}$ to the basin of infinity $A_{Q}=\left\{z: Q^{n}(z) \rightarrow \infty\right.$ as $\left.n \rightarrow \infty\right\}$, and $J_{Q}=\partial A_{Q}=$ $\partial K_{Q}$ is the Julia set (here and below $Q^{n}(z)$ is the image of $z$ by the $n$-iterate $Q^{n}$ of $Q$ for $n$ non-negative and the full preimage of $z$ by $Q^{|n|}$ for $n$ negative).

Let $u_{Q}: A_{Q} \rightarrow \mathbb{R}_{+}$be Green's function in $A_{Q}$ such that $u_{Q}(z) \sim \log |z|+$ $o(1)$ as $z \rightarrow \infty$. For all $z$ in some neighborhood $W$ of $\infty, u_{Q}(z)=\log \left|B_{Q}(z)\right|$

2020 Mathematics Subject Classification: 37F10, 37F20, 37F25.

Key words and phrases: Julia set, renormalization, external rays.

Received 29 January 2021; revised 17 February 2021.

Published online 9 March 2021. 
where $B_{Q}$ is the Böttcher coordinate of $Q$ at $\infty$, i.e., a univalent function from $W$ onto $\{w:|w|>R\}$, for some $R>1$, such that $B_{Q}(Q(z))=B_{Q}(z)^{\operatorname{deg} Q}$ for $z \in W$ and $B_{Q}(z) / z \rightarrow 1$ as $z \rightarrow \infty$.

An equipotential of $Q$ of level $b>0$ is the level set $\left\{z: u_{Q}(z)=b\right\}$. Alternatively, the equipotential containing a point $z \in A_{Q}$ is the closure of the union $\bigcup_{n>0} Q^{-n}\left(Q^{n}(z)\right)$ and $u_{Q}(z)=\lim _{n \rightarrow \infty}(\operatorname{deg}(Q))^{-n} \log \left|Q^{n}(z)\right|$ is the level of this equipotential where $b=u_{Q}(z)$ is called the $Q$-level of $z \in A_{Q}$. Note that $u_{Q}(Q(z))=(\operatorname{deg} Q) u_{Q}(z)$ for all $z \in A_{Q}$.

The gradient flow for Green's function (potential) $u_{Q}$ equipped with direction from $\infty$ to $J_{Q}$ defines $Q$-external rays. More specifically, the gradient flow has singularities precisely at the critical points of $u_{Q}$ which are preimages by $Q^{n}, n=0,1, \ldots$, of critical points of $Q$ that lie in the basin of infinity $A_{Q}$. If a trajectory $R$ of the flow that starts at $\infty$ does not meet a critical point of $u_{Q}$, it extends as a smooth (analytic) curve, external ray $R$, up to $J_{Q}$. If $R$ does meet a critical point of $u_{Q}$, one should consider instead two corresponding (non-smooth) left and right external rays as left and right limits of smooth external rays tending to $R$ (for a visualization of such rays, see e.g., Figures 1(a-b) of [LP96] or images in [PZ19]-[PZ20]; to get an impression about the geometry of the Julia set of renormalizable polynomials, see e.g. the computer images of [Pict]). Each external ray $R$ is parameterized by the level of equipotential $b \in(+\infty, 0)$.

The argument $\tau \in \mathbb{T}:=\mathbb{R} / \mathbb{Z}$ of an external ray $R$ is the argument of the curve $R$ asymptotically at $\infty$. Informally, $\tau$ is the argument at which $R$ crosses the "circle at infinity". The correspondence between external rays and their arguments is one-to-one on smooth rays and two-to-one on nonsmooth ones. If $R$ is a $Q$-external ray of argument $\tau$ then $Q(R)$ is also a ray of argument $\sigma_{\operatorname{deg}(Q)}(\tau)$ where $\sigma_{k}(t)=t k(\bmod 1)$. Note that, for any $b$ large enough, $B_{Q}$ maps the equiponential of level $b$ onto the round circle $\left\{|w|=e^{b}\right\}$ and arcs of external rays from this equipotential to $\infty$ onto standard rays that are orthogonal to this circle. Finally, $K_{Q}$ is connected if and only if $B_{Q}$ extends as a univalent function to the basin of infinity $A_{Q}$, if and only if all external rays of $Q$ are smooth.

Let $\mathbb{S}=\{|z|=1\}$ be the unit circle which we identify - when this is not confusing — with $\mathbb{T}$ via the exponential $\mathbb{T} \ni t \mapsto \exp (2 \pi i t) \in \mathbb{S}$.

1.2. Polynomial-like maps and renormalization. Let us recall [DH2] that a triple $\left(W, W_{1}, f\right)$ is a polynomial-like map if $W, W_{1}$ are topological discs, $\overline{W_{1}} \subset W$ and $f: W_{1} \rightarrow W$ is a proper holomorphic map of some degree $m \geq 2$. The set of non-escaping points $K_{f}=\bigcap_{n=1}^{\infty} f^{-n}(W)$ is called the filled Julia set of $\left(W, W_{1}, f\right)$. By the Straightening Theorem [DH2], there exists a monic centered polynomial $G$ of degree $m$ which is hybrid equivalent to $f$, i.e., there is a quasiconformal homeomorphism $h: \mathbb{C} \rightarrow \mathbb{C}$ which is 
conformal a.e. on $K_{f}$, such that $G \circ h=h \circ f$ near $K_{f}$. The map $h$ is called a straightening. This implies in particular that $K_{f}$ is the set of limit points of $\bigcup_{n>0} f^{-n}(z)$ for any $z \in W$ with, perhaps, at most one exception.

We say that another polynomial-like map $\left(\tilde{W}, \tilde{W}_{1}, \tilde{f}\right)$ of the same degree $m$ is equivalent to $\left(W, W_{1}, f\right)$ if there is a component $E$ of $W \cap \tilde{W}$ such that $K_{f} \subset E$ and $f=\tilde{f}$ in a neighborhood of $K_{f}$. Taking a point $z$ as above close to $J_{f}=\partial K_{f}$, it follows (cf. [McM, Theorem 5.11]) that $K_{f}=K_{\tilde{f}}$ and that this is indeed an equivalence relation for polynomial-like maps. Denote by $\mathbf{f}$ the equivalence class of the polynomial-like map $\left(W, W_{1}, f\right)$, by $K_{\mathbf{f}}, J_{\mathbf{f}}$ the corresponding filled Julia set and Julia set of (any representative of) $\mathbf{f}$, and by $f$ the restriction to a neighborhood of $K_{\mathbf{f}}$ of an f-representative (i.e., for any two representatives $\left(W^{(i)}, W_{1}^{(i)}, f_{i}\right), i=1,2$, we have $f_{1}=f_{2}=f$ in a neighborhood of $K_{\mathbf{f}}$ ).

From now on, let us fix a monic centered polynomial $P: \mathbb{C} \rightarrow \mathbb{C}$ of degree $d>1$.

We say that $\mathbf{f}$ is a renormalization of $P$ (cf. [McM], [Inou]) if $\mathbf{f}$ is an equivalence class of polynomial-like maps such that $K_{\mathrm{f}}$ is a connected proper subset of $K_{P}$ and, for some $r \geq 1, f=P^{r}$ in a neighborhood of $K_{\mathbf{f}}$.

1.3. Assumptions. Suppose that

(p1) $\mathbf{f}$ is a renormalization of $P$.

To avoid a situation when an external ray of $P$ can have a limit point in $J_{\mathbf{f}}$ as well as a limit point off $J_{\mathbf{f}}$, we introduce another condition:

(p2) There exists a representative $\left(W^{*}, W_{1}^{*}, f\right)$ of the renormalization $\mathbf{f}$ of $P$ and some $b_{*}>0$ as follows. If $z \in \partial W_{1}^{*}$ belongs to an external ray of $P$ which has a limit point in $K_{\mathbf{f}}$ then the P-level of $z$ is at least $b_{*}$, i.e., $u_{P}(z) \geq b_{*}$.

Let us stress that external rays of $P$ as in (p2) can cross the boundaries of $W^{*}, W_{1}^{*}$ many times (or e.g. have joint arcs with the boundaries).

This condition holds if $W^{*}$ is obtained by the following frequently used construction that we only indicate here; see [Mil1, McM], Inou for details. In the first step, a simply connected domain $W_{0}$ is built using an appropriate Yoccoz puzzle so that $\partial W_{0}=L_{\text {hor }} \cup L_{\text {vert }} \cup F$ where $L_{\text {hor }}$ is a union of finitely many arcs of a fixed equipotential of $P, L_{\text {vert }}$ is a union of finitely many arcs of external rays of $P$ between ends of arcs of $L_{\mathrm{hor}}$, and $F$ is a finite set of repelling periodic points of $J_{P}$ or/and their preimages such that $K_{\mathbf{f}} \subset W_{0} \cup F$ and $f: f^{-1}\left(W_{0}\right) \rightarrow W_{0}$ is a branched covering. By the construction, every external ray of $P$ to $J_{\mathbf{f}} \backslash F$ must cross the "horizontal" part $L_{\text {hor }}$ so that (p2) is obviously satisfied for the set of those rays. If either $L_{\text {vert }}=F=\emptyset$ (as in Example 1 that follows) or $F \cap K_{\mathbf{f}}=\emptyset$, one can take $W^{*}=W_{0}$ so that 
(p2) holds for $W_{1}^{*}=f^{-1}\left(W^{*}\right)$. If $F \subset J_{\mathbf{f}}$, then $W_{0} \backslash f^{-1}\left(W_{0}\right)$ is a degenerate annulus. Then, in the second step, $W^{*}$ is modified from $W_{0}$ by "thickening" [Mil1, p. 12] around points of the set $F$, which adds only finitely many rays (tending to $F$ ). Then (p2) holds for $W_{1}^{*}=f^{-1}\left(W^{*}\right)$ as well.

Example 1. Assume that the Julia set of the polynomial $P$ is disconnected and $K$ is a component of $K_{P}$ different from a point. In this case $K=K_{\mathbf{f}}$ for some renormalization $\mathbf{f}$ of $P$ and conditions (p1)-(p2) are fulfilled. The boundary of $W^{*}$ (hence of $W_{1}^{*}$, too) can be chosen to be merely a component of an equipotential that encloses $K$. With such a choice, each intersection point of an external ray of $P$ with $\partial W^{*}$ has a fixed level so every external ray can cross the boundaries of $W^{*}$ and $W_{1}^{*}$ at most once.

Our goal is to study a correspondence between external rays of $P$ that have limit points in $J_{\mathbf{f}}$, on the one hand, and external, or polynomial-like rays of the renormalization $\mathbf{f}$, on the other (up to a change of straightening, see below). In the case of disconnected Julia set $J_{P}$ and the renormalization $\mathbf{f}$ as in Example 1 this has been done in [LP96], [ABC16, Sect. 6], and [PZ19].

1.4. Polynomial-like rays. For a curve $\alpha:[0,1) \rightarrow \overline{\mathbb{C}}$, the limit (or principal, or accumulation) set of $\alpha$ is $\operatorname{Pr}(\alpha)=\bar{\alpha} \backslash \alpha$.

Let us define external rays of the renormalization $\mathbf{f}$. By [DH2], since $K_{\mathbf{f}}$ is connected, the monic centered polynomial $G$ of degree $m$ which is hybrid equivalent to any representative of $\mathbf{f}$ is uniquely defined by $\mathbf{f}$. Let $h$ be a straightening of $\mathbf{f}$. By this we mean a quasiconformal homeomorphism $\mathbb{C} \rightarrow \mathbb{C}$ which is conformal a.e. on $K_{\mathbf{f}}$ and satisfies $G \circ h=h \circ f$ on some neighborhood of $K_{\mathbf{f}}$. One can also assume that $h$ is conformal at $\infty$ such that $h^{\prime}(\infty) \neq 0$.

As the filled Julia set $K_{G}$ is connected, given $t \in \mathbb{T}$ there is a unique external ray of $G$ of argument $t$, denoted by $R_{t, G}$. Its $h^{-1}$-image $l_{t}^{h}:=h^{-1}\left(R_{t, G}\right)$ is called the polynomial-like ray to $K_{\mathbf{f}}$ of argument $t$. As $h: \mathbb{C} \rightarrow \mathbb{C}$ is a homeomorphism, $\operatorname{Pr}\left(l_{t}^{h}\right)=h^{-1}\left(\operatorname{Pr}\left(R_{t, G}\right)\right)$. Note that the straightening $h$ is not unique. However, the polynomial $G$ is unique, and if $\tilde{h}$ is another straightening, although $\tilde{h}$ defines another system of polynomial-like rays, the homeomorphism $\tilde{h}^{-1} \circ h: \mathbb{C} \rightarrow \mathbb{C}$ maps $l_{t}^{h}$ onto $l_{t}^{\tilde{h}}$ and $\operatorname{Pr}\left(l_{t}^{h}\right)$ onto $\operatorname{Pr}\left(l_{t}^{\tilde{h}}\right)$ ).

In what follows we fix a straightening map $h: \mathbb{C} \rightarrow \mathbb{C}$ (see Theorem 3 (e) and its proof though). Then the set $\left\{l_{t}\right\}$ of polynomial-like rays is fixed, too (where we omit $h$ in $l_{t}^{h}$ as $h$ is fixed). For brevity, $P$-external rays are called $P$-rays, or just rays, and polynomial-like rays to $K_{\mathrm{f}}$ are $f$-rays, or polynomial-like rays.

1.5. Main results. Given a connected compact set $K \subset \mathbb{C}$ which is different from a point, we say that a curve $\gamma:[0,1) \rightarrow \Omega:=\mathbb{C} \backslash K$ converges to a prime end $\hat{P}$ of $K$ if, for a conformal homeomorphism $\psi: \mathbb{C} \backslash K \rightarrow\{|z|>1\}$, 
the curve $\psi \circ \gamma:[0,1) \rightarrow\{|z|>1\}$ converges to a single point $P \in \mathbb{S}$; we say that $\gamma$ converges to the prime end $\hat{P}$ non-tangentially if moreover $\psi \circ \gamma$ converges to the point $P$ non-tangentially, i.e., the set $\psi \circ \gamma((1-\epsilon, 1))$ lies inside a sector (Stolz angle) $\{z:|\arg (z-P)-\arg P| \leq \alpha\}$ for some $\epsilon>0$ and $\alpha \in(0, \pi / 2)$. Furthermore, we say that two curves $\gamma_{1}, \gamma_{2}:[0,1) \rightarrow \Omega$ are $K$-equivalent if they both converge to the same prime end and moreover have the same limit sets $\operatorname{Pr}\left(\gamma_{1}\right)=\operatorname{Pr}\left(\gamma_{2}\right)$ in $\left.\partial K{ }^{1}\right)$. By Lindelöf's theorem (see e.g. [Pom, Theorem 2.16]), if two curves converge to the same prime end non-tangentially, they share the same limit set. Therefore, if $\gamma_{1}, \gamma_{2}$ converge to the same prime end of $K$ non-tangentially, then $\gamma_{1}, \gamma_{2}$ are also $K$-equivalent.

The following statement was proved in [ABC16] $\left[\left(^{2}\right)\right.$ in the set up of Example 1.

Theorem 1 (cf. [ABC16, Theorem 6.9]). Assume (p1)-(p2) hold. For each $P$-ray $R$ that has an accumulation point in $K_{\mathbf{f}}$ we have $\operatorname{Pr}(R) \subset J_{\mathbf{f}}$ and there is a unique polynomial-like ray $l=\lambda(R)$ such that the curves $l$, $R$ are $K_{\mathbf{f}}$-equivalent. Moreover, $l, R$ converge to a single prime end of $K_{\mathbf{f}}$ non-tangentially. Furthermore, $\lambda: R \mapsto l$ maps the set of $P$-rays to $K_{\mathbf{f}}$ onto the set of polynomial-like rays, and is "almost injective": $\lambda$ is one-to-one except when one and only one of the following (i)-(ii) holds. Suppose that $\lambda^{-1}(\ell)=\left\{R_{1}, \ldots, R_{k}\right\}$ with $k>1$.

(i) $k=2$ and both rays $R_{1}, R_{2}$ are non-smooth and share a common arc starting at a critical point of Green's function $u_{P}$ to $J_{\mathbf{f}}$, or

(ii) there is $z \in J_{\mathbf{f}}$ such that $\operatorname{Pr}\left(R_{i}\right)=\{z\}, i=1, \ldots, k$, at least two of the rays $R_{1}, \ldots, R_{k}$ are disjoint, and, for some $n \geq 0, P^{r n}(z) \in Y$ where $Y \subset J_{\mathbf{f}}$ is a finite collection of repelling or parabolic periodic points of $P$ that depends merely on $K_{\mathbf{f}}$.

If $K_{P}$ is connected then (i) is not possible.

Note that in case (ii) any two disjoint $P$-rays completed by the joint limit point $z$ split the plane into two domains such that one of them contains $K_{\mathbf{f}} \backslash\{z\}$, and the other one, points from $K_{P} \backslash K_{\mathbf{f}}$. In particular, if $K_{P}$ is connected, the second domain must contain a component of $K_{P} \backslash K_{\mathbf{f}}$ that goes all the way to a pre-periodic point $z \in J_{\mathbf{f}}$. In fact, this is "if and only if": see Theorem 2(b) below.

For an illustration, see e.g. pictures in [McM, p. 116, explained in Example IV, p. 115] of a "dragon" filled Julia set of a quadratic polynomial $P$ admitting three renormalizations; the maps $\lambda$ corresponding to theses renormalizations are one-to-one except at countably many polynomial-like rays

$\left({ }^{1}\right)$ One can show that if $\gamma_{1}$ converges to a single point $a \in \partial K$, then $\gamma_{2}$ is $K$-equivalent to $\gamma_{1}$ if and only if $\gamma_{1}, \gamma_{2}$ are homotopic through a family of curves in $\Omega$ converging to $a$.

$\left({ }^{2}\right)$ In $\mathrm{ABC16}$, a different terminology is used. 
where $\lambda$ is 6 -to- 1 in the top picture, 2 -to- 1 in the left bottom and 3 -to- 1 in the right bottom. In all three cases, the landing points of rays where $\lambda$ is not one-to-one are (pre-)periodic to a fixed point of $P$ where six $P$-rays land.

The next two theorems are consequences of the proof of Theorem 1 .

Theorem 2. Assume (p1)-(p2).

(a) If a point $a \in J_{\mathbf{f}}$ is accessible along a curve $s$ in $\mathbb{C} \backslash K_{\mathbf{f}}$, then $a$ is the landing point of a $P$-ray $R$; moreover the curves $s, R$ are $K_{\mathbf{f}}$-equivalent.

(b) There exists a finite set $Y \subset J_{\mathbf{f}}$ of repelling or parabolic periodic points of $f$, as follows. Let $S$ be a component of $K_{P} \backslash K_{\mathbf{f}}$ such that $(\bar{S} \backslash S) \cap J_{\mathbf{f}}$ $\neq \emptyset$. Then $\bar{S} \backslash S$ is a single point $b \in J_{\mathbf{f}}$, and moreover $f^{n}(b) \in Y$ for some $n \geq 0$.

Note that part (a) is in fact an easy corollary of Lemma 2.1 similar to a result of [LP96]. Part (b) is void if (and only if) $K_{\mathbf{f}}$ is itself a component of $K_{P}$.

For the next statement, we introduce the following notations. Let $\Lambda \subset \mathbb{T}$ be the set of arguments of all $P$-rays that have their limit points in $J_{\mathbf{f}}$. Observe that by Theorem 1 the whole limit sets of such rays are in $J_{\mathbf{f}}$ and, given $\tau \in \Lambda$, there is a unique $P$-ray, denoted by $R_{\tau, P}$, which has its limit set in $J_{\mathbf{f}}$. Indeed, this is obvious if the $P$-ray of argument $\tau$ is smooth. On the other hand, if there are two $P$-rays, left and right, of argument $\tau$, only one of them can have its limit point in $J_{\mathbf{f}}$ because the other one must go to another component of $K_{P}$. Now, the map $\lambda$ of Theorem 1 induces a map $p: \Lambda \rightarrow \mathbb{T}$ such that for all $\tau \in \Lambda$,

$$
\lambda\left(R_{\tau, P}\right)=l_{p(\tau)} .
$$

By Theorem 1, $\operatorname{Pr}\left(l_{p(\tau)}\right)=\operatorname{Pr}\left(R_{\tau, P}\right)$, and moreover $R_{\tau, P}, l_{p(\tau)}$ are $K_{\mathbf{f}}$-equivalent.

Given a positive integer $k$, let $\sigma_{k}: \mathbb{T} \rightarrow \mathbb{T}, \sigma_{k}(t)=k t(\bmod 1)$. Recall that $\operatorname{deg}(f)=m$. Let $D:=\operatorname{deg}\left(P^{r}\right)=d^{r}$.

Theorem 3 (cf. [PZ19]).

(a) $\Lambda$ is a compact nowhere dense subset of $\mathbb{T}$ which is invariant under $\sigma_{D}$.

(b) $\sigma_{m} \circ p=p \circ \sigma_{D}$ on $\Lambda$.

(c) The map $p: \Lambda \rightarrow \mathbb{T}$ is surjective and finite-to-one, and moreover "almost injective" as defined in Theorem 1 .

(d) $p: \Lambda \rightarrow \mathbb{T}$ extends to a continuous monotone degree one map $\tilde{p}: \mathbb{T} \rightarrow \mathbb{T}$.

(e) The map $p$ is unique in the following sense: if $\tilde{p}: \Lambda \rightarrow \mathbb{T}$ corresponds to another straightening $\tilde{h}$, then $\tilde{p}(t)=p(t)+k /(m-1)(\bmod 1)$ for some $k=0,1, \ldots, m-1$.

In the set up of Example 1 i.e., when $K_{P}$ is disconnected and $K_{f}$ is a periodic component of $K_{P}$, Theorem 3 was proved in [PZ19] (by a different 
method), with part (c) replaced by an explicit bound for the cardinality of fibers of the map $p$ as well as with an extra statement about the Hausdorff dimension of the set $\Lambda$.

A detailed proof of the main Theorem 11 is given in Sect. 2 and the proofs of Theorems 2 3 are in Sect. 3 . The proof of Theorem 1 follows rather closely the proofs of [LP96, Lemma 2.1] and [ABC16, Theorems 6.8-6.9]. An essential difference is that we have to adapt the proofs to the situation that external rays of $P$ can cross the boundary of $W_{1}^{*}$ as in (p2) many times.

2. Proof of Theorem 1, Let $f: W_{1}^{*} \rightarrow W^{*}$ be a representative of $\mathbf{f}$ as in (p2). As $K_{f}$ is connected, all the critical points of $f$ are in $K_{f}$. Hence, for each $k, f^{k}: f^{-k}\left(W_{1}^{*} \backslash K_{f}\right) \rightarrow W_{1}^{*} \backslash K_{f}$ is an unbranched (degree $\left.m^{k}\right)$ map. Therefore, $L_{k}:=f^{-k}\left(\partial W_{1}^{*}\right)$ is the boundary of a simply connected domain $f^{-k}\left(W_{1}^{*}\right)$.

Let $\mathcal{R}$ denote the set of all $P$-rays $R$ such that $R$ has a limit point in $J_{f}$. First, we show that all limit points of $R \in \mathcal{R}$ are in $J_{f}$, introducing some notations along the way. Let

$$
b_{*, k}=\inf \left\{u_{P}(z): z \in R \cap L_{k}, R \in \mathcal{R}\right\} .
$$

By (p2), $b_{*, 0}>0$. As $R \in \mathcal{R}$ implies $P^{r}(R) \in \mathcal{R}$, we have $b_{*, k} \geq b_{*, 0} / D^{k}$, hence $b_{*, k}>0$, for all $k$. Let $R \in \mathcal{R}$ and $k \geq 0$. Since $R \cap L_{k}$ is a closed set and $b_{*, k}>0$, there exists a unique point $z_{k}(R) \in R \cap L_{k}$ such that $u_{P}\left(z_{k}(R)\right)=\inf \left\{u_{P}(z): z \in R \cap L_{k}\right\}$. Observe that the $\operatorname{arc} \Gamma_{k, R}$ of $R$ from $z_{k}(R)$ down to $J_{P}$ lies entirely in $\overline{f^{-k}\left(W_{1}^{*}\right)}$. As $\bigcap_{k \geq 0} \overline{f^{-k}\left(W_{1}^{*}\right)}=K_{f}$, we see immediately that the limit set of $R$, which is $\bigcap_{k>0} \overline{\Gamma_{k, R}}$, is a subset of $J_{f}$.

Before proceeding with more notations and the main lemma, let us note that $b_{*, k}=b_{*, 0} / D^{k}, k=1,2, \ldots$ Indeed, as $f^{k}: f^{-k}\left(W_{1}^{*} \backslash K_{f}\right) \rightarrow W_{1}^{*} \backslash K_{f}$ is an unbranched covering, each component of $f^{-k}(R)$ is an arc of some ray from $\mathcal{R}$. This implies that $b_{*, k} \leq b_{*, 0} / D^{k}$. The opposite inequality was seen before.

Now, choose a conformal isomorphism $\psi$ from $\mathbf{C} \backslash K_{f}$ onto $\mathbf{D}^{*}=\{|z|>1\}$ such that $\psi(z) / z \rightarrow e$ as $z \rightarrow \infty$, for some $e>0$. A curve $\tilde{R}$ in $\mathbf{D}^{*}$ with limit set in $\mathbb{S}=\{|z|=1\}$ is called a $K$-related ray if its preimage $\psi^{-1}(\tilde{R})$ is a $P$-ray $R \in \mathcal{R}$, i.e., $R$ has its limit set in $K_{f}$. The argument of $\tilde{R}$ is said to be the argument of the ray $\psi^{-1}(\tilde{R})$. Let $A_{K}=\psi\left(W^{*} \backslash K_{f}\right)$ be an "annulus" with boundary curves $\psi\left(\partial W^{*}\right)$ and $\mathbb{S}$. Denote $\tilde{z}_{k}(\tilde{R})=\psi\left(z_{k}(R)\right.$. Note that $\tilde{z}_{k}(\tilde{R}) \in \psi\left(L_{k}\right) \cap \tilde{R}$ and the arc of the $R$-related ray $\tilde{R}$ from $\tilde{z}_{k}(\tilde{R})$ to $\mathbb{S}$ is contained in the "annulus" between $\psi\left(L_{k}\right)$ and $\mathbb{S}$. An arc of a $K$-related ray $\tilde{R}=\psi(R)$ from the point $\tilde{z}_{0}(\tilde{R})=\psi\left(z_{0}(R)\right) \in \psi\left(L_{0}\right)$ to $\mathbb{S}$ is called a $K$-related arc. Its argument is the argument of the corresponding ray. The following main lemma and its proof are minor adaptations of the ones of [LP96, Lemma 2.1]. 
LEMMA 2.1.

$1^{\circ}$ Every $K$-related arc has a finite length, and hence converges to a unique point of $\mathbb{S}$.

$2^{\circ}$ For every closed arc $I \subset \mathbb{S}$ (in particular a point), the set $K(I)$ of arguments of all $K$-related arcs converging to a point of $I$ is a non-empty compact set.

$3^{\circ}$ The set of all $K$-related arcs in $\{z: 1<|z|<1+\epsilon\}$ converging to a point $z_{0}$ lies in a Stolz angle

$$
\left\{z:\left|\arg \left(z-z_{0}\right)-\arg z_{0}\right| \leq \alpha\right\},
$$

where $\alpha \in(0, \pi / 2)$ and $\epsilon$ do not depend on $z_{0} \in \mathbb{S}$.

Proof. $1^{\circ}$ Let $B_{*, k}=\sup \left\{u_{P}(z): z \in L_{k}\right\}$. For every $k \geq 0$ there is a number $C_{k}$ such that, for every ray $R \in \mathcal{R}$, the length of the $\operatorname{arc} R_{k}$ of $R$ between the points $z_{k}(R)$ and $z_{k+1}(R)$ is bounded by $C_{k}$. This is because the latter arc is an arc of a $P$-ray that joins two equipotentials of positive levels $B_{*, k}, b_{*, k}$. Denote $\tilde{L}_{k}=\psi\left(L_{k}\right)$. Then $\tilde{L}_{k}$ is a compact subset of $A_{K}$ which surrounds $\mathbb{S}$. By the above, every $K$-related arc $\tilde{R}$ splits into $\operatorname{arcs}$ $\tilde{R}_{k}=\psi\left(R_{k}\right), k \geq 0$, i.e., $\tilde{R}_{k}$ is the arc of $\tilde{R}$ joining $\tilde{z}_{k}(\tilde{R})$ and $\tilde{z}_{k+1}(\tilde{R})$. For every $k$, the supremum of the lengths over all arcs $\tilde{R}_{k}$ of the $K$-related rays $\tilde{R}$ is bounded by

$$
\tilde{C}_{k}=C_{k} \sup \left\{\left|\psi^{\prime}(z)\right|: z \in \bar{W}_{1}^{*} \backslash f^{-k-2}\left(W_{1}^{*}\right)\right\} .
$$

Let $A_{1, K}=\psi\left(W_{1}^{*} \backslash K_{f}\right)$ and $g=\psi \circ f \circ \psi^{-1}: A_{1, K} \rightarrow A_{K}$. Then $z$ tends to $\mathbb{S}$ if and only if $g(z)$ tends to $\mathbb{S}$. It is well-known (see e.g. [P86]) that $g$ extends to an expanding holomorphic map in an annulus $U_{0}=\{z$ : $\left.1-\rho_{0}<|z|<1+\rho_{0}\right\}$ for some $\rho_{0}>0$. This means that after passing if necessary to an iterate of $g$ (which we also denote $g$ ) we have

$$
\left|\left(g^{-1}\right)^{\prime}(z)\right|<c<1
$$

for every $z \in U_{0}$ and for every branch $g^{-1}$ such that $g^{-1}(z) \in U_{0}$.

Fix a set $\tilde{L}_{m} \subset U=A_{K} \cap U_{0}$ for some $m$ large enough. Then, for each $n=1,2, \ldots, \tilde{L}_{n+m}=\left\{z \in U: g^{n}(z) \in \tilde{L}_{n+m}\right\}$. Denote by $l_{n}$ the supremum of the lengths of $\tilde{R}_{n+m}$ over all $R \in \mathcal{R}$. Note that each $l_{n}$ is finite, because $l_{n} \leq \tilde{C}_{m+n}$. In fact, much more is true: as $g^{n}\left(\tilde{R}_{n+m}\right)$ is $\tilde{S}_{m}$ for some ray $S \in \mathcal{R}$, 11 yields $l_{n}<c^{n} l_{0}$. Given a $K$-related ray $\tilde{R}$, the length of its arc from the point $\tilde{z}_{m}(\tilde{R})$ to $\mathbb{S}$, which is in the component of $\mathbf{C} \backslash \tilde{\gamma}_{0}$ containing $\mathbb{S}$, is bounded from above by $\sum_{n=0}^{\infty} c^{n} l_{0}<\infty$. Moreover, the same argument shows the following

CLAim 1. The lengths of the arcs of K-related rays $\tilde{R}$ between $\tilde{z}_{k}(\tilde{R})$ and $\mathbb{S}$ tend uniformly to zero (exponentially in $k$ ). 
$2^{\circ}$ Fix a closed non-degenerate $\operatorname{arc} I \subset \mathbb{S}$. There exists a $K$-related ray converging to a point of $I$. Indeed, otherwise no $K$-related ray ends in the arc $g^{n}(I)$, for any $n$. This is impossible because $g^{n}(I)=\mathbb{S}$ for large $n$ and the set of $K$-related rays is non-empty (for example, it contains images by $\psi$ of $P$-rays landing at repelling periodic points of the polynomial-like map $f: W_{1}^{*} \rightarrow W^{*}$; for the existence of such $P$-rays, see [Mil0, [EL89], [LP96]). We need to show that the set $K(I)$ of arguments of all $K$-related rays ending in $I$ is closed.

This is an immediate consequence of the next claim which follows, basically, from Claim 1 and will also be useful later on. Given a $K$-related ray $\tilde{R}_{t}$ of argument $t$ (i.e., $t \in \Lambda$ ) consider its arc $\hat{r}_{t}$ between $\tilde{L}_{0}$ and $\mathbb{S}$, parameterized as a curve $\tilde{r}_{t}:\left[b_{*, 0}, 0\right] \rightarrow A_{K} \cup \mathbb{S}$ as follows. For any $b \in\left[b_{*, 0}, 0\right)$, define the point $r_{t}(x) \in A_{K}$ to be such that $\psi^{-1}\left(r_{t}(x)\right)$ is a point of a $P$-ray of argument $t$ and equipotential level $b$. Finally, let $\tilde{r}_{t}(0)=\lim _{b \rightarrow 0} \tilde{r}_{t}(x) \in \mathbb{S}$ where the limit exists by $1^{\circ}$.

Claim 2. The family $\tilde{\mathcal{R}}=\left\{\tilde{r}_{t}\right\}_{t \in \Lambda}$ is a compact subset of $C\left[b_{*, 0}, 0\right]$.

Let us first show that this family is equicontinuous. In view of Claim 1 , this will follow from the equicontinuity of the restricted family $\tilde{\mathcal{R}}_{m}=\left\{\hat{r}_{t}\right.$ : $\left.\left[b_{*, 0}, b_{*, 0} / D^{m}\right] \rightarrow A_{K}\right\}$ for each integer $m>1$. Fix $m$ and consider two objects: a compact set $E_{m} \subset \mathbb{C}$ bounded by the equipotential of levels $b_{*, 0}$ and $b_{*, 0} / D^{m}$ of $P$ and a family $\mathcal{R}_{m}$ of (closed) arcs in $E_{m}$ of all $P$-rays that join the equipotential levels $b_{*, 0}, b_{*, 0} / D^{m}$ and are parameterized by the equipotential level $b \in\left[b_{*, 0}, b_{*, 0} / D^{m}\right]$. It is easy to see that this is a compact subset of $C\left[b_{*, 0}, b_{*, 0} / D^{m}\right]$ (indeed, map this family by a fixed high iterate of $P$ to a family of smooth arcs of $P$-rays which are preimages of segments of standard rays by the Böttcher coordinate $B_{P}$ at infinity; hence, this new family is compact; then pull it back). As $\mathcal{R}_{m} \subset C\left[b_{*, 0}, b_{*, 0} / D^{m}\right]$ is compact, it is equicontinuous. In turn, since $\psi^{-1}$ is a homeomorphism on $E_{m}$ (onto its image) and each $\psi^{-1}\left(\tilde{r}_{t}\right)$ is in $\mathcal{R}_{m}$, the family $\tilde{\mathcal{R}}_{m}$ is equicontinuous too. Thus $\tilde{\mathcal{R}}$ is an equicontinuous family.

It remains to prove that it is closed. So suppose a sequence $\hat{r}_{t_{n}}$ converges uniformly in $\left[b_{*, 0}, 0\right]$. In particular, $\hat{r}_{t_{n}}$ crosses $\tilde{L}_{k}$ for each $k$ large enough. One can assume that $t_{n}$ tends to some $t$. Then the sequence of $\operatorname{arcs}$ of $P$-rays $\psi^{-1} \circ \tilde{r}_{t_{n}}$, on the one hand, tends, uniformly on each interval $\left[b_{*, 0}, b_{*, 0} / D^{m}\right]$, to an arc $r$ of a $P$-ray of argument $t$, on the other hand, crosses each $L_{k}$ with $k$ large. Hence, $r$ has a limit point in $K_{f}$. Applying $\psi$ we find that the limit of $\tilde{r}_{t_{n}}$ is a $K$-related arc, which ends the proof of the claim.

This proves $2^{\circ}$ when $I$ is not a single point. By the intersection of compacta, $2^{\circ}$ also holds if $I$ is a point.

$3^{\circ}$ Every branch of $g^{-n}$ is a well defined univalent function in every disc contained in $U_{0}$. Hence, by the Koebe distortion theorem (see e.g. [Gol]), one 
can choose $0<\rho^{\prime}<\rho_{0}$ such that for every

$$
z \in U^{\prime}=\left\{z: 1-\rho^{\prime}<|z|<1+\rho^{\prime}\right\},
$$

every $n=1,2, \ldots$ and every branch $g^{-n}$,

$$
\left|\frac{\left(g^{-n}\right)^{\prime}(x)}{\left(g^{-n}\right)^{\prime}(y)}\right|<2
$$

whenever $|z-x|<\rho^{\prime}$ and $|z-y|<\rho^{\prime}$.

We reduce $U^{\prime}$ further as follows. By Claim 1, fix $m_{0}>m$ such that the length of the arc of any $K$-related ray $\tilde{R}$ between $\tilde{z}_{m_{0}}(\tilde{R})$ and $\mathbb{S}$ is less than $\rho^{\prime}$. On the other hand, if $z$ lies in an unbounded component of $R \backslash z_{m_{0}}(R)$, i.e., in the arc of $R$ between $z_{m_{0}}(R)$ and $\infty$, then $u_{P}(z) \geq b_{*, m_{0}}$, in particular, there is $r>0$ independent of $z$ and $R$ as above such that the distance between $z$ and $J_{P}$ is at least $r$. Therefore, there exists some $\rho_{1} \in\left(0, \rho^{\prime}\right)$ such that for every $z \in\left\{z: 1<|z|<1+\rho_{1}\right\}$, if $z$ belongs to a $K$-related ray $\tilde{R}$ then $z$ lies in an arc of $\tilde{R}$ between $\tilde{z}_{m_{0}}(\tilde{R})$ and $\mathbb{S}$. Let

$$
U_{1}=\left\{z: 1-\rho_{1}<|z|<1+\rho_{1}\right\} .
$$

We introduce the following notations:

Given $x \in U_{1}$, denote by $l_{x}$ the part of the $K$-related ray passing through $x$ between $x$ and $\mathbb{S}$ (if such a ray exists). This notation is correct: as already noted before, if another $K$-related ray passes through $x$ and next ramifies from $l_{x}$, it goes to a component of $\psi(J(f))$, not to $\mathbb{S}$. So it is not $K$-related.

Denote by $h_{x}$ the interval which joins $x$ and $\mathbb{S}$, orthogonal to $\mathbb{S}$. Denote by $l(x)$ and $h(x)$ the corresponding Euclidean lengths. Find a large enough $N$ such that $\tilde{\gamma}_{0}:=\tilde{L}_{N}$ in $U_{1}$. By the choice of $U_{1}$,

$$
l(x)<\rho^{\prime} \quad \text { for all } x \text { between } \tilde{\gamma}_{0} \text { and } \mathbb{S} .
$$

Let $\tilde{\gamma}_{1}=g^{-1}\left(\tilde{\gamma}_{0}\right)$. There exists a positive $\beta_{0}$ less than 1 such that

$$
\frac{h(x)}{l(x)}>\beta_{0}
$$

for all points $x$ in the annulus $V$ between $\tilde{\gamma}_{0}$ and $\tilde{\gamma}_{1}$.

Fix the maximal $\epsilon_{0}>0$ such that

$$
U_{2}=\left\{z: 1-\epsilon_{0}<|z|<1+\epsilon_{0}\right\}
$$

does not intersect $\tilde{\gamma}_{1}$. We intend to prove assertion $3^{\circ}$ of our lemma with

$$
\alpha=\arccos \left(\frac{\beta_{0}}{8 L}\right)
$$

where $L=\sup \left\{\left|g^{\prime}(z)\right|: z \in U_{0}\right\}$ and with $\epsilon$ between 0 and $\epsilon_{0}$ so small that $1<|z|<1+\epsilon$ and $h(z) /\left|z-z_{0}\right| \geq 2 \cos \alpha$ implies $\left|\arg \left(z-z_{0}\right)-\arg z_{0}\right| \leq \alpha$. 
It is enough to prove that

$$
\frac{h(x)}{l(x)}>\beta=\frac{\beta_{0}}{4 L}
$$

for all $x \in U_{2}$. Assume the contrary: there exists $x_{*} \in U_{2}$ that belongs to some $K$-related ray $\tilde{R}$ with

$$
h\left(x_{*}\right) / l\left(x_{*}\right) \leq \beta .
$$

Choose the minimal $n \geq 1$ such that $g^{n}\left(x_{*}\right) \in V$.

The lengths $h^{(i)}$ and $l^{(i)}$ of the curves $g^{i}\left(h_{x_{*}}\right)$ and $g^{i}\left(l_{x_{*}}\right)$ cannot exceed $\rho^{\prime}$ for all $i=0,1, \ldots, n$. This holds for $l^{(i)}$ by $(3)$, because $g^{i}\left(x_{*}\right)$ is between $\tilde{\gamma}_{0}$ and $\mathbb{S}$. We cope with $h^{(i)}$ 's by induction: Length $\left(h^{(0)}\right)<\rho$ by the definition of $U_{1}$. If it holds for all $i \leq j-1$ then by 2 ,

$$
\frac{h^{(j-1)}}{l^{(j-1)}} \leq 4 \beta=\beta_{0} / L \text {. }
$$

Then

$$
h^{(j)} \leq L h^{(j-1)} \leq \beta_{0} \cdot l^{(j-1)}<l^{(j-1)}<\rho^{\prime} .
$$

Now we use the assumption (6) and again apply (2) to obtain, for $z_{*}=$ $g^{n}\left(x_{*}\right) \in \tilde{S}_{N}$,

$$
\frac{h\left(z_{*}\right)}{l\left(z_{*}\right)} \leq \frac{h^{(n)}}{l^{(n)}} \leq 4 \beta=\beta_{0} / L<\beta_{0} .
$$

This contradicts (4).

Comment. The key bound (5) can also be seen directly from (4) (with, for instance, $\beta=\beta_{0} / 10$ ) by applying, besides the Koebe distortion bound (2), another distortion bound: there is a function $\epsilon:(0,1) \rightarrow(0,+\infty)$, with $\epsilon(r) \rightarrow 0$ as $r \rightarrow 0$, such that for any univalent function $\varphi$ on the unit disc, if $\varphi(0)=0$ and $\varphi^{\prime}(0)=1$, then

$$
\left|\log \frac{\varphi(z)}{z}\right|<\epsilon(|z|)
$$

(see e.g. Gol]). This bound is applied to the function

$$
\varphi(z)=\frac{g^{-n}\left(w+\rho_{0} z\right)-g^{-n}(w)}{\left(g^{-n}\right)^{\prime}(w) \rho_{0}}
$$

where $n$ is minimal such that $g^{n}(x) \in V$, and $w \in \mathbb{S}$ is the projection of $g^{n}(x)$ to $\mathbb{S}$ and reducing $\rho^{\prime}$. Note that $\left(g^{-n}\right)^{\prime}(w)>0$ because $g$ preserves $\mathbb{S}$.

We continue as follows (cf. [ABC16, proof of Theorem 6.9]). Recall that a straightening $h: \mathbb{C} \rightarrow \mathbb{C}$ is a quasiconformal homeomorphism which is holomorphic at $\infty$ and $h^{\prime}(\infty) \neq 0$. It conjugates the polynomial-like map $f$ to the polynomial $G$ near their filled Julia sets $K_{f}$ and $K_{G}$. Let $B_{G}: A_{G} \rightarrow \mathbb{D}^{*}$ be the Böttcher coordinate of $G$ such that $B_{G}(z) / z \rightarrow 1$ as $z \rightarrow \infty$, which is well defined in the basin of infinity $A_{G}=\mathbb{C} \backslash K_{G}$ as $K_{G}$ is connected. 
We have the following picture:

$$
\mathbb{D}^{*} \stackrel{\psi^{-1}}{\longrightarrow} \mathbb{C} \backslash K_{f} \stackrel{h}{\rightarrow} \mathbb{C} \backslash K_{G} \stackrel{B_{G}}{\longrightarrow} \mathbb{D}^{*} .
$$

Consider the map $\Psi:=\psi \circ h^{-1} \circ B_{G}^{-1}: \mathbb{D}^{*} \rightarrow \mathbb{D}^{*}$ from the uniformization plane of the polynomial $G$ to the $g$-plane of $K$-related rays. It is a quasiconformal homeomorphism which is holomorphic at $\infty$. For $u \in \mathbb{S}$, let $L_{u}=\Psi\left(r_{u} \cap \mathbb{D}^{*}\right)$ where $r_{u}=\{t u: t>0\}$ is a standard ray in the uniformization plane of $G\left({ }^{3}\right)$.

LEMma 2.2. The curve $L_{u}$ converges non-tangentially to a unique point $z_{0}=z_{0}(u)$ of the unit circle $\mathbb{S}$. Moreover, there is $\beta \in(0, \pi / 2)$ such that, for any $u \in \mathbb{S}$ and all $z \in L_{u}$ close enough to $\mathbb{S}$,

$$
\left|\arg \left(z-z_{0}\right)-\arg z_{0}\right| \leq \beta .
$$

Here $\beta$ depends only on the quasiconformal deformation of the straightening map $h$. Furthermore, for every $z_{0} \in \mathbb{S}$ there exists a unique $u$ such that $L_{u}$ lands at $z_{0}$.

Proof of Lemma 2.2 (cf. [ABC16, Section 6]). The map $\Psi: \mathbb{D}^{*} \rightarrow \mathbb{D}^{*}$ extends to a homeomorphism of the closures and then to a quasiconformal homeomorphism $\Psi^{*}$ of $\mathbb{C}$ by $\overline{\Psi^{*}(z)}=1 / \Psi^{*}(1 / \bar{z})$ (see [Ahl]). Note that the quasiconformal deformations of $\Psi$ and $\Psi^{*}$ are the same, equal to the quasiconformal deformation $M$ of the straightening map $h$. Consider the curve $L_{u}^{*}=\Psi^{*}\left(r_{u}\right)$. It is an extension of the curve $L_{u}$, which crosses $\mathbb{S}$ at a point $z_{0}=\Psi^{*}(u)$. As a quasiconformal image of a straight line, the curve $L_{u}^{*}$ has the following property [Ahl]: there exists $C=C(M)>0$ such that

$$
\left|z-z_{0}\right| /|z-1 / \bar{z}|<C \quad \text { for every } z \in L_{u}^{*} \text {. }
$$

Therefore, $L_{u}^{*}$ tends to $z_{0}$ non-tangentially; moreover, (8) holds for some $\beta=\beta(C(M))$. The last claim follows from the fact that $\Psi^{*}$ is a homeomorphism.

Now, define the correspondence $\lambda$ as follows (having in mind (7)). Let $R$ be a $P$-ray to $K_{f}$. By Lemma 2.1 , the $K$-related ray $\tilde{R}=\psi(R)$ tends to a point $z_{0} \in \mathbb{S}$. By Lemma 2.2, there exists a unique $L_{u}$ which tends to $z_{0}$. The curve $\psi^{-1}\left(L_{u}\right)=h^{-1} \circ B_{G}^{-1}(\{t u: t>1\})$ is a polynomial-like ray $l_{\tau}$ where $u=e^{2 \pi i \tau}$. Let

$$
\lambda(R):=\psi^{-1}\left(L_{u}\right) .
$$

The correspondence $\lambda$ is "onto" by the first claim of Lemma 2.2 along with Lemma 2.1 $\left(2^{\circ}\right)$.

Now, both curves $\tilde{R}, L_{u}$ in $\mathbb{D}^{*}$ tend to the point $z_{0} \in \mathbb{S}$ non-tangentially, by Lemmas 2.1 and 2.2 respectively. Then, by definition, the $P$-ray $R$ and the

$\left({ }^{3}\right)$ Note that the curve $L_{u}$ lies in the left-hand disc $\mathbb{D}^{*}$ of $\sqrt{7}$ while the point $u$ is at the boundary of the right-hand disc there. 
polynomial-like ray $\lambda(R)$ converge to a single prime end of $K_{\mathbf{f}}$ non-tangentially, hence $R$ and $\lambda(R)$ are also $K_{\mathbf{f}}$-equivalent. Finally, the condition that $R$ and $\lambda(R)$ are $K_{\mathbf{f}}$-equivalent uniquely determines the polynomial-like ray $\lambda(R)$.

It remains to prove the "almost injectivity" of $\lambda$. This is a direct consequence of the one-to-one correspondence between $K$-related rays and curves $L_{u}$ established above and the following claim whose proof is identical to the one of [ABC16, Theorem 6.8] (for completeness, we reproduce it below with obvious changes in notation). While passing from $K$-related rays to $P$-rays we use the fact that if a $K$-related ray is periodic, the corresponding $P$-ray converges to a periodic point of $P$ which is either repelling or parabolic (by the Snail Lemma [Mil0], it cannot be irrationally indifferent).

LEMma 2.3. Any point $w \in \mathbb{S}$ is the landing point of precisely one $K$ related ray, except when one and only one of the following holds:

(i) $w$ is the landing point of exactly two $K$-related rays which are non-smooth and have a common smooth arc that goes to $w$;

(ii) $w$ is a landing point of at least two disjoint $K$-related rays, in which case $w$ is a (pre)periodic point of $g$ and some iterate $g^{n}(w)$ belongs to a

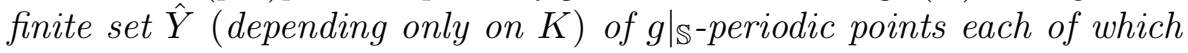
is the landing point of finitely many, but at least two, $K$-related rays, which are periodic of the same period depending merely on the landing point $w(4)$.

Moreover, if $w$ is periodic then (i) cannot hold.

Proof. Assume that there are two $K$-related rays landing at a point $w \in \mathbb{S}$ and that (i) does not hold. We need to prove that then (ii) holds. Since (i) does not hold, there exist disjoint $K$-related rays landing at $w$. Let us study this case in detail.

Associate to any such pair of rays $\hat{R}_{t}, \hat{R}_{t^{\prime}}$ an open arc $\left(\hat{R}_{t}, \hat{R}_{t^{\prime}}\right)$ of $\mathbb{S}$ as follows. Two points of $\mathbb{S}^{1}$ with the arguments $t, t^{\prime}$ split $\mathbb{S}$ into two arcs. Let $\left(\hat{R}_{t}, \hat{R}_{t^{\prime}}\right)$ be the one that contains no arguments of $K$-related rays except possibly for those that land at $w$. Geometrically, this means the following. The $K$-related rays $\hat{R}_{t}, \hat{R}_{t^{\prime}}$ together with $w \in \mathbb{S}$ split the plane into two domains. The arc $\left(\hat{R}_{t}, \hat{R}_{t^{\prime}}\right)$ corresponds to one of them, disjoint from $\mathbb{S}$. Let $L\left(\hat{R}_{t}, \hat{R}_{t^{\prime}}\right)=\delta$ be the angular length of $\left(\hat{R}_{t}, \hat{R}_{t^{\prime}}\right)$. Clearly, $0<\delta<1$. Now we make a few observations.

(1) If $K$-related disjoint rays of arguments $t_{1}, t_{1}^{\prime}$ land at a common point $w_{1}$ while $K$-related disjoint rays of arguments $t_{2}, t_{2}^{\prime}$ land at a point $w_{2} \neq w_{1}$, then the arcs $\left(\hat{R}_{t_{1}}, \hat{R}_{t_{1}^{\prime}}\right),\left(\hat{R}_{t_{2}}, \hat{R}_{t_{2}^{\prime}}\right)$ are disjoint.

$\left({ }^{4}\right)$ In [ABC16, Theorem 6.8(ii)], it is claimed erroneously that all $K$-related rays to the point $w$ are smooth (cf. [PZ20]). Note that this claim is not relevant to the rest of ABC16. 
The above follows from the definition of the arc $\left(\hat{R}_{t}, \hat{R}_{t}\right)$.

(2) If disjoint $K$-related rays $\hat{R}_{t}, \hat{R}_{t^{\prime}}$ of arguments $t, t^{\prime}$ land at a common point $w$, then the $K$-related rays $g\left(\hat{R}_{t}\right), g\left(\hat{R}_{t^{\prime}}\right)$ are also disjoint and land at the common point $g(w)$. Moreover,

$$
L\left(g\left(\hat{R}_{t}\right), g\left(\hat{R}_{t^{\prime}}\right)\right) \geq \min \{D \delta(\bmod 1), 1-D \delta(\bmod 1)\}>0 .
$$

Indeed, the images $g\left(\hat{R}_{t}\right), g\left(\hat{R}_{t^{\prime}}\right)$ are disjoint near $g(w)$, because $g$ is locally one-to-one. Hence, $g\left(\hat{R}_{t}\right) \cap g\left(\hat{R}_{t^{\prime}}\right)=\emptyset$, because otherwise the corresponding $P$-rays would have their limit sets in different components of $K_{P}$, a contradiction since both rays $g\left(\hat{R}_{t}\right), g\left(\hat{R}_{t^{\prime}}\right)$ are $K$-related. Since the argument of $g\left(\hat{R}_{t}\right)$ is represented by the point $D t(\bmod 1) \in(0,1)$, we get the inequality of $(2)$.

Let us consider the following set $\hat{Z}(K)$ of points in $\mathbb{S}: w \in \hat{Z}(K)$ if and only if there is a pair of disjoint $K$-related rays $\hat{R}, \hat{R}^{\prime}$ which both land at $w$ and satisfy $L\left(\hat{R}, \hat{R}^{\prime}\right) \geq 1 /(2 D)$. Denote by $\hat{Y}(K)$ the set of periodic points which are in forward images of the points of $\hat{Z}(K)$.

(3) If the set $\hat{Z}(K)$ is non-empty, then it is finite, and consists of (pre)periodic points.

Indeed, $\hat{Z}(K)$ is finite by (1). Assume $w \in \hat{Z}(K)$. Then by (2) some iterate $g^{n}(w)$ must hit $\hat{Z}(K)$ again.

To complete the proof, choose disjoint $K$-related rays $\hat{R}_{t}, \hat{R}_{t^{\prime}}$ landing at $w \in \mathbb{S}$ and use this to prove that all claims of (ii) hold.

We show that the orbit $w, g(w), \ldots$ cannot be infinite. Indeed, otherwise by (1)-(2), we have a sequence of non-degenerate pairwise disjoint arcs $\left(g^{n}\left(\hat{R}_{t}\right), g^{n}\left(\hat{R}_{t^{\prime}}\right)\right) \subset \mathbb{S}, n=0,1, \ldots$ By $(2)$, some iterates of $w$ must hit the finite set $\hat{Z}(K)$ and hence $\hat{Y}(K)$ (which are therefore non-empty), a contradiction.

Hence for some $0 \leq n<l, g^{n}(w)=g^{l}(w)$; let us verify that the other claims of (ii) hold. Replacing $w$ by $g^{n}(w)$, we may assume that $w$ is a (repelling) periodic point of $g$ of period $k=l-n$. By (2), $w \in \hat{Y}(K)$. By [LP96. Theorem 1], the set of $K$-related rays landing at $w$ is finite, and each $K$-related ray landing at $w$ is periodic with the same period. Hence, (ii) holds. Finally, the last claim of the lemma follows because a periodic non-smooth ray must have infinitely many broken points, hence, no other ray can have a common arc with it that goes up to the Julia set; see $\mathrm{ABC16}$, Lemma 6.1] for details.

\section{Proofs of Theorems 2}

3.1. Theorem 2, Part (a) is an immediate corollary of Lemma 2.1 and Lindelöf's theorem, as in [LP96]. Indeed, since a curve $s \subset W \backslash K_{\mathbf{f}}$ converges 
to a point $a \in K_{\mathbf{f}}$, the curve $\tilde{s}=\psi(s)$ converges to a point $z_{0} \in \mathbb{S}$, and the limit of the function $\psi^{-1}$ along the curve $\tilde{s}$ exists and equals $a$. By Lemma 2.1. there is a $K$-related ray $\tilde{R}$ that tends to $z_{0}$, and it tends non-tangentially. Then, by [Pom, Corollary 2.17], the $P$-ray $R$ converges to the same point $a$. By definition, the curves $s, R$ are $K_{\mathbf{f}}$-equivalent.

Let us prove part (b). The closed set $S \cup K_{f}$ is connected and so too is its complement (by the Maximum Principle). Consider the set $\hat{S}=\psi(S) \subset \mathbb{D}^{*}$. Let $I=\overline{\hat{S}} \backslash \hat{S}$. Then $I$ is a connected closed subset of the unit circle $\mathbb{S}$.

Let us prove $I$ is a single point. Otherwise there is an interior point $x \in I$ which is $g$-periodic. Let $\beta$ be a $K$-related ray that lands at $x$. Notice that since $x$ is an interior point of $I, \beta$ must cross $\hat{S}$. Now, since $x$ is $g$-periodic, $R=\psi^{-1}(\beta)$ is a periodic $P$-ray, hence it converges to a periodic point $a \in \bar{S} \backslash S$ of $P$ and crosses $S$, a contradiction since $S \subset K_{P}$. This proves that $I$ is a single point; denote it by $z_{0}$.

Choose two sequences $z_{n}^{\prime}, z_{n}^{\prime \prime}$ of $\mathbb{S}$ tending to $z_{0}$ from the left and from the right respectively, and two sequences of $K$-related rays $l_{n}^{\prime}, l_{n}^{\prime \prime}$ so that $l_{n}^{\prime}$ lands at $z_{n}^{\prime}$ and $l_{n}^{\prime \prime}$ lands at $z_{n}^{\prime \prime}$. Then, passing perhaps to subsequences, by Claim 2 in the proof of Lemma 2.1. the sequence $l_{n}^{\prime}$ tends to a $K$-related ray $l^{\prime}$ and $l_{n}^{\prime \prime}$ tends to a $K$-related ray $l^{\prime \prime}$, where $l^{\prime}$ and $l^{\prime \prime}$ land at the same $z_{0}$. By the above, $l^{\prime}, l^{\prime \prime}$ are disjoint.

Now we apply Lemma 2.3 to conclude that $z_{0}$ is $g$-(pre-)periodic, and some iterate of $z_{0}$ lies in a finite set $\hat{Y} \subset \mathbb{S}$ of periodic points, which is independent of $z_{0}$. Hence, the point $a$ is $P$-(pre-)periodic, and some iterate of $a$ lies in a finite set $Y \subset J_{f}$ of periodic points, which is independent of $a$. As every point of $Y$ is a landing point of a periodic ray, it can be either repelling or parabolic.

3.2. Theorem 3. Proof of (b), (c): It follows from the definition of $\Lambda$ that $\sigma_{D}(\Lambda)=\Lambda$ and $\sigma_{m} \circ p=p \circ \sigma_{D}$ on $\Lambda$. By invariance and since $\Lambda \neq \mathbb{T}$, the set $\Lambda$ contains no intervals; (c) is a reformulation of a part of the statement of Theorem 1.

Proof of (a), (d): Considering $\Lambda$ as a subset of $\mathbb{S}=\{|z|=1\}$ define a new map $p_{K}: \Lambda \rightarrow \mathbb{S}$ as follows: for $\tau \in \Lambda$, let $p_{K}(\tau) \in \mathbb{S}$ be the landing point of a $K$-related ray of argument $\tau$. Recall the map $\Psi=\psi \circ h^{-1} \circ B_{G}^{-1}: \mathbb{D}^{*} \rightarrow \mathbb{D}^{*}$ introduced in the proof of Theorem 1, and its quasi-conformal extension $\Psi^{*}: \mathbb{C} \rightarrow \mathbb{C}$. By Lemma 2.2 and the definition of the maps $\lambda$ and $p$, we have

$$
p_{K}=\left.\Psi^{*}\right|_{\mathbb{S}} \circ p \text {. }
$$

Since $\Psi^{*}: \mathbb{S} \rightarrow \mathbb{S}$ is an orientation preserving homeomorphism, it is enough to prove (a), (d) with $p$ replaced by $p_{K}$. By Lemma $2\left(2^{\circ}\right), p_{K}^{-1}(I)$ is closed in $\mathbb{S}$ for any closed arc $I \subset \mathbb{S}$. Therefore, $\Lambda=p_{K}^{-1}(\mathbb{S})$ is closed and the map $p_{K}: \Lambda \rightarrow \mathbb{S}$ is continuous. To show (d), define an extension $\tilde{p}_{K}: \mathbb{S} \rightarrow \mathbb{S}$ of 
$p_{K}: \Lambda \rightarrow \mathbb{S}$ in an obvious way as follows. Let $J:=\left(t_{1}, t_{2}\right)$ be a component of $\mathbb{S} \backslash \Lambda$. Then $p_{K}\left(t_{1}\right)=p_{K}\left(t_{2}\right)=: w_{J}$ because otherwise there would be a point of $\mathbb{S}$ with no $K$-related rays landing at it. Let $\tilde{p}_{K}(\tau)=w_{J}$ for all $\tau \in J$. Then $\tilde{p}_{K}: \mathbb{S} \rightarrow \mathbb{S}$ is continuous. Now, given $t \in \mathbb{S}$, the set $\tilde{p}_{K}^{-1}(\{t\})$ is either a singleton or a non-trivial closed arc. This follows from the definition of $\tilde{p}_{K}$ and because $K$-related rays with different arguments do not intersect unless case (i) of Theorem 2.3 takes place. Therefore, $\tilde{p}_{K}: \mathbb{S} \rightarrow \mathbb{S}$ is monotone and of degree one.

Proof of (e): Let $\tilde{h}$ be another straightening, $\tilde{\Psi}: \mathbb{D}^{*} \rightarrow \mathbb{D}^{*}$ the corresponding quasiconformal map and $\tilde{\Psi}^{*}: \mathbb{C} \rightarrow \mathbb{C}$ its quasiconformal extension. As $p_{K}: \mathbb{S} \rightarrow \mathbb{S}$ is independent of the straightening, by $(9)$ we have $\tilde{p}=\left.T\right|_{\mathbb{S}} \circ p$ where $T=\left(\tilde{\Psi}^{*}\right)^{-1} \circ \Psi^{*}$. On the other hand, on $\mathbb{D}^{*}, T=\left(B_{G} \circ \tilde{h}\right) \circ\left(B_{G} \circ h\right)^{-1}$, hence $T$ commutes with $z \mapsto z^{m}$ for $|z|>1$ near $\mathbb{S}$, by definitions of $h, B_{G}$. Therefore, the homeomorphism $\nu:=\left.T\right|_{\mathbb{S}}: \mathbb{S} \rightarrow \mathbb{S}$ commutes with $z \mapsto z^{m}$ on $\mathbb{S}$, too. It is then well known that $\nu(z)=v z$ for some $v \in \mathbb{C}$ with modulus 1 such that $v^{m}=v$ (proof: as $\nu(1)^{m}=\nu(1)$ let $v=\nu(1)$, so that a homeomorphism $\nu_{0}=v^{-1} \nu: \mathbb{S} \rightarrow \mathbb{S}$ commutes with $z \mapsto z^{m}$ too and $\nu_{0}(1)=1$; then there is a lift $\tilde{\nu}_{0}: \mathbb{R} \rightarrow \mathbb{R}$ of $\nu_{0}$ such that $\tilde{\nu}_{0}(0)=0, \tilde{\nu}_{0}-1$ is 1-periodic and $\tilde{\nu}_{0}(m x)=m \tilde{\nu}_{0}(x)$ for all $x \in \mathbb{R}$, which in turn implies $\tilde{\nu}_{0}\left(n / m^{k}\right)=n / m^{k}$ for all $n, k \in \mathbb{Z}_{>0}$; by continuity, $\tilde{\nu}_{0}(x)=x$ for all $\left.x\right)$.

Acknowledgments. In [Le12, we answered, under an extra assumption, a question posed by Alexander Blokh to the author whether an accessible point of the filled Julia set $K_{f}$ of a renormalization $f$ of $P$ by some curve outside of $K_{f}$ is always accessible by an external ray of $P$ (i.e., by a curve outside of the filled Julia set $K_{P}$ ). Theorem 2(a) strengthens this result of [Le12], under a weaker assumption (p2). Theorem 3 was added following a recent work [PZ19] which also served as an inspiration for writing up this paper. Finally, we would like to thank Feliks Przytycki for a helpful discussion and the referee for comments that helped to improve the exposition.

\section{References}

[Ahl] L. Ahlfors, Lectures on Quasiconformal Mappings, Van Nostrand, Princeton, 1966.

[ABC16] A. Blokh, D. Childers, G. Levin, L. Oversteegen and D. Schleicher, An extended Fatou-Shishikura inequality and wandering branch continua for polynomials, Adv. Math. 228 (2016) 1121-1174.

[CG] L. Carleson and Th. W. Gamelin, Complex Dynamics, Springer, 1993.

[DH1] A. Douady and J. H. Hubbard, Exploring the Mandelbrot Set. The Orsay Notes, 1983-1984, preprint.

[DH2] A. Douady and J. H. Hubbard, On the dynamics of polynomial-like mappings, Ann. Sci. École Norm. Sup. (4) 18 (1985), 287-343. 
[EL89] A. Eremenko and G. Levin, Periodic points of polynomials, Ukrainian Math. J. 41 (1989), 1258-1262.

[Gol] G. M. Goluzin, Geometric Theory of Functions of a Complex Variable., Transl. Math. Monogr. 26, Amer. Math. Soc., 1969.

[Inou] H. Inou, Renormalization and rigidity of polynomials of higher degree, J. Math. Kyoto Univ. 42 (2002), 351-392.

[LS91] G. Levin and M. L. Sodin, Polynomials with disconnected Julia sets and Green maps, preprint 23/1990-91, Landau Center for Research in Mathematical Analysis, Inst. Math., The Hebrew Univ. of Jerusalem, 1991; https://www.researchgate.net/publication/317411781.

[Le12] G. Levin, Rays to renormalizations, manuscript, 2012.

[LP96] G. Levin and F. Przytycki, External rays to periodic points, Israel J. Math. 94 (1996), 29-57.

[McM] C. McMullen, Complex Dynamics and Renormalization, Ann. of Math. Stud. 135, Princeton Univ. Press, 1994.

[Mil0] J. Milnor, Dynamics in One Complex Variable: Introductory Lectures, Springer, 2000 .

[Mil1] J. Milnor, Local connectivity of Julia sets: expository lectures, arXiv:math/9207220 (1992).

[PZ19] C. Petersen and S. Zakeri, On the correspondence of external rays under renormalization, arXiv:1903.00800 (2019).

[PZ20] C. Petersen and S. Zakeri, Periodic points and smooth rays, arXiv:2009.02788 (2020).

[Pict] http://people.math.harvard.edu/ ${ }^{\sim}$ ctm/gallery/julia/feig.html.

[Pom] Ch. Pommerenke, Boundary Behavior of Conformal Maps, Springer, 1992.

[P86] F. Przytycki, Riemann map and holomorphic dynamics, Invent. Math. 85 (1986), 439-455.

Genadi Levin

Institute of Mathematics

The Hebrew University of Jerusalem

Givat Ram, Jerusalem, 91904, Israel

ORCID: 0000-0003-0763-4711

E-mail: genady.levin@mail.huji.ac.il 
